Proceedings of the 40th lEEE

Conference on Decision and Control

Orlando, Florida USA, December 2001

\title{
WeM02-3
}

\section{Constrained output regulation of discrete-time linear plants}

\author{
Guoyong Shi*
}

\author{
Ali Saberi*
}

Anton A. Stoorvogel ${ }^{\dagger}$

\author{
Peddapullaiah Sannuti ${ }^{\ddagger}$
}

\begin{abstract}
Output regulation of discrete-time linear systems with state and/or input constraints on magnitude is considered. The structural properties of linear plants are identified under which the so called constrained semiglobal and global output regulation problems are solvable. As in the case of continuous-time systems, an important aspect of our development is a taxonomy of constraints to show a clear relationship between the type of constraints and the output regulation results. Solvability conditions are developed for semiglobal and global output regulation problems. Appropriate regulators are constructed.
\end{abstract}

\section{Introduction}

Output regulation is one of the most important and widely studied problems in control systems. Pioneering works are found in [2-6]. Recently, problems of output regulation with constraints have received much attention $[8,13]$. A recent book [11] formulates and studies a number of problems on different facets of output regulation of linear systems with or without input constraints. However, most of the works focus on the regulation with only input constraints.

Having studied during the last decade several aspects of control design problems for linear systems with magnitude and rate constraints on control variables, during the last two years the research thrust of the last three authors and their students has broadened to include magnitude constraints on control variables as well as state variables. Recent work $[7,9,10]$ considered linear systems not only with input magnitude constraints but with state magnitude constraints as well. In particular, $[9,10]$ consider internal stabilization while [7] consider output regulation in different frameworks, namely global, semiglobal, and regional frameworks. One of the fundamental aspects developed in all these works is a taxonomy of constraints yielding a clear relationship between the type of constraints and what can or cannot be achieved. Then, the solvability conditions for the posed problems are identified in terms of such a taxonomy of constraints. Regarding output regulation, the work in [7] deals with continuous-time linear systems. Our interest in this paper is to study output regulation for discrete-time systems with both state as well as input magnitude constraints. Although the development for discrete-time systems parallels somewhat the results for continuous-time systems, discrete-time systems distinguish themselves from continuous-time systems in anumber of ways; (1) the solvability conditions for the posed problems differ from those of continuous-time systems, (2) techniques of proofs are different, and (3) the methods of constructing appropriate regulators need to be revised as well.

Owing to lack of space, the proofs of the results developed here are omited in this conference version of the paper.

Our notations are mostly standard. We use $k$ as the discrete time index and $x^{+}$to indicate the time shift, i.e. $x^{+}(k)=x(k+1)$. Also, we let $\mathbb{C}, \mathbb{C}^{\oplus}, \mathbb{C}^{\ominus}$ and $\mathbb{C}^{0}$ denote respectively the set of complex numbers in the entire complex plane, outside the unit circle, inside the unit circle, and on the unit circle.

\section{Preliminaries and Problem Formulation}

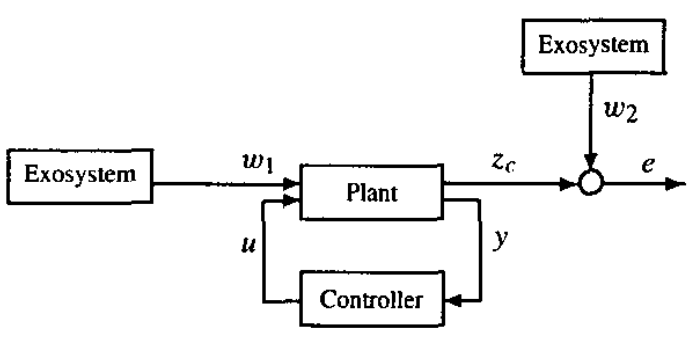

Output regulation consists of two objectives: asymptotic tracking and asymptotic disturbance rejection. Consider the setup of Figure 2.1. One exosystem generates a reference signal $w_{2}$, and the control objective is to make the controlled output $z_{c}$ track this reference signal so that the tracking error signal $e(k)=z_{c}(k)-w_{2}(k) \rightarrow 0$ as $k \rightarrow \infty$. The other exosystem models and generates an

\footnotetext{
${ }^{*}$ School of Electrical Eng. and Comp. Science, Washington State University, Pullman, WA 99164-2752, U.S.A., E-mail: \{gshi,saberi\} @eecs.wsu.edu. The work of Ali Saberi and Guoyong Shi is partially supported by the National Science Foundation under Grant ECS-0000475.

$\ddagger$ Department of Mathematics and Computing Science, Eindhoven Univ. of Technology, P.O. Box 513, 5600 MB Eindhoven and Department of Information Technology and Systems, Delft Univ. of Technology, P.O. Box 5031, 2600 GA Delft. The Netherlands, E-mail: a.a.stoorvogel@tue.nl

${ }^{\ddagger}$ Department of Electrical and Computer Engineering, Rutgers University, 94 Brett Road, Piscataway, NJ 08854-8058, U.S.A., E-mail: sannuti@ece.rutgers.edu
} 
exogenous disturbance $w_{1}$ that acts on the system, and the associated control objective is to reduce the effect of the disturbance $w_{1}$ on the output $z_{r}$.

By combining the two exosystems into one large exosystem we can transform the block diagram in Figure 2.1 into the block diagram of Figure 2.2 where the additional output $z$ is to be explained shortly.

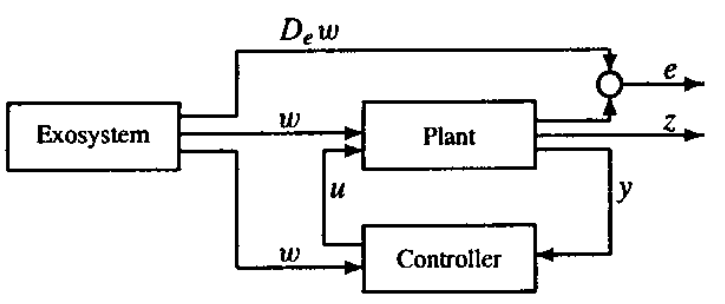

The dynamic equations of the linear time-invariant system in Figure 2.2 are

$$
\Sigma:\left\{\begin{array}{rlrl}
x^{+} & =A x+B u+E w, & x \in \mathbb{R}^{n}, u \in \mathbb{R}^{m} \\
w^{+} & =S w & & w \in \mathbb{R}^{s} \\
y & =C_{y} x+D_{y} w & & y \in \mathbb{R}^{\ell} \\
e & =C_{e} x+D_{e} w & & e \in \mathbb{R}^{r} \\
z & =C_{z} x+D_{z} u & & z \in \mathbb{R}^{p}
\end{array}\right.
$$

where $y$ is the measurement output, and $e$ represents the output regulation error. The second equation describes the exosystem, which models the class of reference signals and/or exogenous disturbances. It generates $E w$ which is a disturbance affecting the system and $D_{y} w$ a disturbance affecting the measurements. But it also generates $-D_{e} w$ which is a reference signal we want to track with the output $C_{e} x$. All the constraints imposed on the plant manifest themselves in the constrained output $z$, which is required to remain in a convex set $\$$. Finally, without loss of generality we assume that $\left(\begin{array}{lll}C_{z} & D_{z}\end{array}\right)$ is surjective throughout this paper.

Our objectives are to achieve: 1) internal stability if the system is free of external signals, i.e. $w=0$, and 2) output regulation, i.e. $e(k) \rightarrow 0$ as $k \rightarrow \infty$. Both objectives are to be attained under the output constraint that $z(k) \in \&$ for all $k \geqslant 0$.

In general, the constraint set $\delta$ discussed in most of the literature is bounded and convex. In fact typically it is a hypercube. Since in most practical problems the constraints on the input $u$ are independent of the value of the state $x$ and vice versa it is reasonable to assume that the constraints on the input and on the states are separable. Therefore, we make the following fundamental assumption on the nature of this constraint set.

Assumption 2.1 The following conditions on \& are satisfied: (i) The set $\&$ is bounded, closed, convex, and contains 0 as an interior point.

(ii) $s=\left(\& \cap \mathrm{im} C_{z}\right) \oplus\left(\& \cap \operatorname{im} D_{z}\right)$.

Remark. Note that the condition (ii) reflects our assumption that the constraints on the input and on the states are separable. Note that Assumption 2.1 actually implies that $D_{z}^{\mathrm{T}} C_{z}=0$.

It is obvious that the initial state of the system must be restricted since we cannot satisfy the constraints if the initial state of the system is arbitrary. For this reason, we define

\section{Definition 2.2 (Admissible set of initial conditions) \\ Let the system (2.3) and the constraint set \& be given. We define}

$$
\chi(\xi):=\left\{x_{0} \in \mathbb{R}^{p} \mid \exists u_{0} \text { such that } C_{z} x_{0}+D_{z} u_{0} \in \&\right\}
$$

as the admissible set of initial conditions.

Remark. In view of part (ii) of Assumption 2.1, we observe that $C_{z} x_{0}+D_{z} u_{0} \in \&$ if and only if $C_{z} x_{0} \in \&$ and $D_{z} u_{0} \in \&$. Since we can always assign $u_{0}$, the admissible set $X(\delta)$ can be equivalently written as

$$
\chi(\xi):=\left\{x_{0} \in \mathbb{R}^{p} \mid C_{z} x_{0} \in \delta\right\} .
$$

Several synthesis problems in the semiglobal or global settings are defined below.

Problem 2.3 Consider the system (2.3), the constraint set $s \subset \mathbb{R}^{p}$, and a bounded set $\boldsymbol{w}_{0} \subset \mathbb{R}^{s}$. The constrained semiglobal state feedback output regulation problem is defined as follows. For any a priori given (arbitrarily large) bounded set $X_{0}$ contained in the interior of $X(\xi)$, find, if possible, a state feedback law (possibly nonlinear) of the form,

$$
\left\{\begin{aligned}
v^{+} & =\ell(x, v, w), \\
u & =g(x, v, w)
\end{aligned} \quad v \in \mathbb{R}^{q}\right.
$$

such that for any a priori given bounded set $\mathcal{V}_{0} \in \mathbb{R}^{q}$, the following conditions hold:

(i) The equilibrium point $(x, v)=(0,0)$ of the closedloop system with $w=0$ is locally exponentially stable with $x_{0} \times v_{0}$ contained in its basin of attraction.

(ii) For any $(x(0), v(0)) \in X_{0} \times \mathcal{V}_{0}$, and $w(0) \in \mathcal{W}_{0}$, we have $z(k) \in \&$ for all $k \geqslant 0$ and $\lim _{k \rightarrow \infty} e(k)=0$.

(iii) For any $(x(0), v(0)) \in X_{0} \times v_{0}$, and $w(0) \in w_{0}$, whenever we set $w(k) \equiv 0$ for $k \geq k_{0}$ for some $k_{0}$, we have $\lim _{k \rightarrow \infty} x(k)=0$. 
Problem 2.4 Consider the system (2.3), the constraint set $s \subset \mathbb{R}^{p}$, and a bounded set $w_{0} \subset \mathbb{R}^{s}$. The constrained global state feedback output regulation problem is defined as follows. Find, if possible, a state feedback law (possibly nonlinear) of the form,

$$
\left\{\begin{aligned}
v^{+} & =\ell(x, v, w), \quad v \in \mathbb{R}^{q} \\
u & =g(x, v, w)
\end{aligned}\right.
$$

such that the following conditions hold:

(i) The equilibrium point $(x, v)=(0,0)$ of the closedloop system is locally exponentially stable with $X(\delta) \times \mathbb{R}^{q}$ contained in its basin of attraction.

(ii) For any $(x(0), v(0)) \in X(\xi) \times \mathbb{R}^{q}$, and $w(0) \in \mathcal{W}_{0}$, we have $z(k) \in s$ for all $k \geqslant 0$, and $\lim _{k \rightarrow \infty} e(k)=0$.

The measurement feedback problem in the global setting is almost never feasible. For this reason we do not formulate the global measurement feedback problem.

Problem 2.5 Consider the system (2.3), the constraint set $s \subset \mathbb{R}^{p}$, and a bounded set $\boldsymbol{w}_{0} \subset \mathbb{R}^{s}$. The constrained semiglobal measurement feedback output regulation problem is defined as follows. For any a priori given (arbitrarily large) bounded set $x_{0}$ contained in the interior of $X(\&)$, find, if possible, a measurement feedback law (possibly nonlinear) of the form,

$$
\left\{\begin{array}{l}
v=\ell(y, v, w), \quad v \in \mathbb{R}^{q} \\
u=g(y, v, w)
\end{array}\right.
$$

such that for any a priori given bounded set $v_{0} \in \mathbb{R}^{q}$, the following conditions hold:

(i) The equilibrium point $(x, v)=(0,0)$ of the closedloop system, is locally exponentially stable with $x_{0} \times \mathcal{V}_{0}$ contained in its basin of attraction.

(ii) For any $(x(0), v(0)) \in x_{0} \times \mathcal{v}_{0}$ and $w(0) \in \mathcal{W}_{0}$, we have $z(k) \in \&$ for all $k \geqslant 0$, and $\lim _{k \rightarrow \infty} e(k)=0$.

(iii) For any $(x(0), v(0)) \in X_{0} \times \mathcal{v}_{0}$, and $w(0) \in w_{0}$, whenever we set $w(k) \equiv 0$ for $k \geq k_{0}$ for some $k_{0}$, we have $\lim _{k \rightarrow \infty} x(k)=0$.

\section{Taxonomy of Constraints}

Let $\Sigma_{z u}$ denote the subsystem characterized by the quadruple $\left(A, B, C_{z}, D_{z}\right)$. It turns out that the structural properties of this subsystem play dominant roles in the study of constrained semiglobal and global output regulation. Specifically, the right invertibility, the location of invariant zeros, and the order of infinite zeros of $\Sigma_{z u}$ determine what can or cannot be achieved. This section is devoted to categorizations of constraints which are to be used in the statements of solvability conditions.

The first categorization is based on whether the subsystem $\Sigma_{z u}$ is right invertible or not. We recall first the definition of right invertibility.

\section{Definition 3.1 (Right invertibility) The system}

$$
\left\{\begin{aligned}
x^{+} & =A x+B u \\
y & =C x+D u
\end{aligned}\right.
$$

where $x \in \mathbb{R}^{n}, u \in \mathbb{R}^{m}$ and $y \in \mathbb{R}^{\ell}$, is said to be right invertible if for any $y_{r e f}(k), k \geqslant 0$, there exists $a u(k)$ and a choice of $x(0)$ such that $y(k)=y_{\text {ref }}(k)$ for all $k \geqslant 0$.

We have the following definition regarding the first categorization of constraints.

Definition 3.2 The constraints are said to be

- right invertible constraints if the subsystem $\Sigma_{z u}$ is right invertible.

- non-right invertible constraints if the subsystem $\Sigma_{z u}$ is non-right invertible.

The second categorization is based on the location of the invariant zeros of the subsystem $\Sigma_{z u}$. We recall next the definition of invariant zeros.

Definition 3.3 (Invariant zeros) The invariant zeros of $a$ linear system with a realization $(A, B, C, D)$ are those points $\lambda \in \mathbb{C}$ for which

$$
\operatorname{rank}\left(\begin{array}{cc}
\lambda I-A & -B \\
C & D
\end{array}\right)<\operatorname{normrank}\left(\begin{array}{cc}
s I-A & -B \\
C & D
\end{array}\right)
$$

where by "normrank" we mean the rank of a matrix with entries in the field of rational functions.

Because of their importance, we specifically label the invariant zeros of the subsystem $\Sigma_{z u}$ as the constraint invariant zeros of the plant.

Definition 3.4 (Constraint invariant zeros) The invariant zeros of the subsystem $\Sigma_{z u}$ are called the constraint invariant zeros of the plant associated with the constrained output $z$.

We have the following definition regarding the second categorization of constraints.

Definition 3.5 The constraints are said to be

- minimum phase constraints if all the constraint invariant zeros are in $\mathbb{C}^{\ominus}$.

- weakly minimum phase constraints if all the constraint invariant zeros are in $\mathbb{C}^{\ominus} \cup \mathbb{C}^{0}$ with the restriction that at least one such constraint invariant zero is in $\mathbb{C}^{0}$ and any such constraint invariant zero in $\mathbb{C}^{0}$ is simple. 
- weakly non-minimum phase constraints if all the constraint invariant zeros are in $\mathbb{C}^{\ominus} \cup \mathbb{C}^{0}$ and at least one constraint invariant zero in $\mathbb{C}^{0}$ is not simple.

- at most weakly non-minimum phase constraints if all the constraint invariant zeros are in $\mathbb{C}^{\ominus} \cup \mathbb{C}^{0}$.

- strongly non-minimum phase constraints if any one or more of the constraint invariant zeros are in $\mathbb{C}^{\oplus}$.

The third categorization is based on the order of the infinite zeros of the subsystem $\Sigma_{z u}$. See [12] for a definition of infinite zeros of a system. Because of their importance, we specifically label the infinite zeros of the subsystem $\Sigma_{z u}$ as the constraint infinite zeros of the plant.

\section{Definition 3.6 (Constraint infinite zeros)}

The infinite zeros of the subsystem $\Sigma_{z u}$ are called the constraint infinite zeros of the plant associated with the constrained output $z$.

We have the following definition regarding the third categorization of constraints.

\section{Definition 3.7 The constraints are said to be}

- type 1 constraints if the order of all constraint infinite zeros is less than or equal to one

- not type 1 constraints if there exist any constraint infinite zeros with order greater than one.

\section{Main Results for Right Invertible Con- straints}

In this section we develop the solvability conditions for the constrained semiglobal and global output regulation problems (Problems 2.3, 2.4, and 2.5) whenever the constraints are right invertible. It is worth noting here that the right invertible constraints include as a special case amplitude and rate constraints on actuators. Whenever we have constraints only on the control variable $u$, we have $z=u$ implying that $C_{z}=0$ and $D_{z}=I_{m}$. In other words, since $\Sigma_{z u}$ characterized by $\left(A, B, 0, l_{m}\right)$ can easily be verified to be right invertible, we note that the amplitude and rate constraints on actuators are indeed right invertible constraints. Owing to lack of space, the development of results under non-right invertible constraints is omited in this conference version of the paper.

In the study of classical output regulation problems without constraints, it is well known that the following standard assumptions are basically necessary:

Assumption 4.1 There exist matrices $\Pi$ and $\Gamma$ satisfying the so-called regulator equation,

$$
\begin{aligned}
\Pi S & =A \Pi+B \Gamma+E \\
0 & =C_{e} \Pi+D_{e} .
\end{aligned}
$$

Assumption 4.2 The matrix $S$ has all its eigenvalues outside the unit circle.

Assumption 4.3 The pair $(A, B)$ is stabilizable.

Assumption 4.4 The pair

$$
\left[\left(\begin{array}{ll}
C_{y} & D_{y}
\end{array}\right),\left(\begin{array}{ll}
A & E \\
0 & S
\end{array}\right)\right]
$$

is observable.

The details for the necessity of these assumptions can be found in [11].

\subsection{Results}

The following theorems provide solvability conditions for the problems posed in Section 2. It turns out that for discrete-time systems the solvability conditions for the semiglobal and global state feedback output regulations are the same. This is in contrast to that in continuous-time systems [7]. The results presented here include the output regulation of linear systems with input constraints as a special case, by specializing $C_{z}=0$ and $D_{z}=I$.

Theorem 4.5 Consider the system (2.3), the constraint set $\& \subset \mathbb{R}^{p}$, and a bounded set $w_{0} \subset \mathbb{R}^{s}$. Assume that the constraints are right invertible, and let Assumptions $4.1,4.2$, and 4.3 be satisfied. Then the constrained global (or semiglobal) state feedback output regulation problem i.e. Problem 2.4 (or respectively Problem 2.3) is solvable if the following conditions hold:

(i) The constraints are at most weakly non-minimum phase.

(ii) The constraint are of type 1 .

(iii) There exists a $\rho \in(0,1)$ such that

$$
\begin{gathered}
\left(C_{z} \Pi+D_{z} \Gamma\right) w(k) \in(1-\rho) s \\
\text { for all } w(k), k \geqslant 0, \text { with } w(0) \in W_{0} .
\end{gathered}
$$

Remark. Conditions (i) and (ii) are necessary for stabilization (see [10]). Condition (iii) is almost necessary as can be seen from the next theorem.

Theorem 4.6 Consider the system (2.3), the constraint set $s \subset \mathbb{R}^{p}$, and a bounded set $w_{0} \subset \mathbb{R}^{s}$. Assume that the constraints are right invertible and let Assumptions 4.1, 4.2 , and 4.3 hold. Under the additional condition that the system from $u$ to $e$ is left-invertible, the constrained global (or semiglobal) state feedback output regulation problem, i.e. Problem 2.4 (or respectively Problem 2.3), for the given set $W_{0}$ is not solvable if there exists $a w$ with $w(0) \in W_{0}$ such that

$$
\left(C_{z} \Pi+D_{z} \Gamma\right) w(k) \notin \&
$$

for some $k>0$. 
We consider next semiglobal output regulation with measurement feedback.

Theorem 4.7 Consider the system (2.3), the constraint set $\& \subset \mathbb{R}^{p}$, and a bounded set $\mathcal{W}_{0} \subset \mathbb{R}^{s}$. Assume that the constraints are right invertible and let Assumptions 4.1, 4.2, 4.3, and 4.4 hold. Then the constrained semiglobal measurement feedback output regulation problem (i.e. Problem 2.5) is solvable if the following conditions hold:

(i) The constraints are at most weakly non-minimum phase.

(ii) The constraint are of type 1 .

(iii) There exists a $\rho \in(0,1)$ such that

$$
\left(C_{z} \Pi+D_{z} \Gamma\right) w(k) \in(1-\rho) \&
$$

for all $w(k), k \geqslant 0$, with $w(0) \in w_{0}$.

(iv) $\operatorname{ker} C_{z} \subset \operatorname{ker} C_{z} A$.

(v) $\operatorname{ker}\left(\begin{array}{ll}C_{y} & D_{y}\end{array}\right) \subset \operatorname{ker}\left(\begin{array}{ll}C_{z} & D_{z}\end{array}\right)$

Remark. It is worth noting that the solvability conditions as given by Theorems 4.5 and 4.7 for right invertible constraints are independent of any specific features or shape of the given constraint set $\$$. However, it will not be so for non-right invertible constraints.

\section{Tracking Problem of Constrained Sys- tems}

When the constraints are strongly non-minimum phase, in general we cannot achieve global or semiglobal stabilization. As we have already seen, output regulation problems are intimately related to internal stabilization problems. Suppose we restrict the output regulation problem to tracking, i.e. assume $E=0$. Then, it is interesting to ask whether one can achieve tracking for any initial conditions in the domain of attraction. This section addresses this tracking problem as depicted in Figure 5.1.

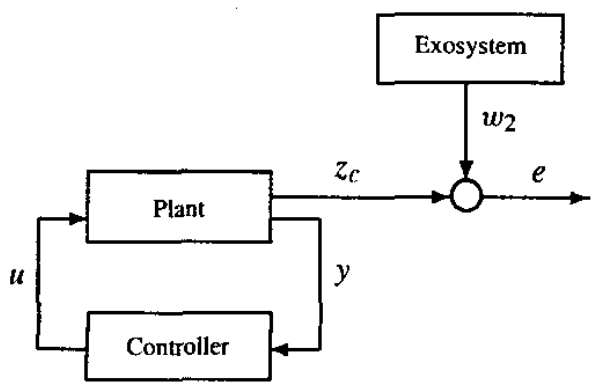

Before we proceed further, the following two notions are needed.
Definition 5.1 (Constrained stabilizing domain) Let $a$ system be given by (2.3) with $w(0)=0$ and let the constraint set $\& \subset \mathbb{R}^{p}$ satisfy Assumption 2.1. The set $\mathcal{P}_{s} \subset \mathcal{X}(\$) \times \mathbb{R}^{4}$ is a constrained stabilizing domain if there exists a state feedback law (possibly nonlinear) of the form,

$$
\left\{\begin{aligned}
v^{+} & =\ell(x, v), \\
u & =g(x, v)
\end{aligned} \quad v \in \mathbb{R}^{q}\right.
$$

such that

(i) For any $(x(0), v(0)) \in \mathcal{P}_{s}$ we have $x(k) \rightarrow 0$ and $v(k) \rightarrow 0$ as $k \rightarrow \infty$.

(ii) For any $(x(0), v(0)) \in \mathcal{P}_{s}$ we have the solution of the closed-loop system satisfying

$$
z(k) \in \& \text { for all } k \geqslant 0 \text {. }
$$

\section{Definition 5.2 (Constrained tracking domain)}

Consider the system (2.3) with a constraint set \& $\subset$ $\mathbb{R}^{p}$ satisfying Assumption 2.1 and a set $\boldsymbol{w}_{0} \subseteq \mathbb{R}^{s}$. The set $\mathcal{P}_{t} \subset X(\$) \times \mathbb{R}^{q}$ is a constrained tracking domain if there exists a state feedback law (possibly nonlinear) of the form,

$$
\left\{\begin{array}{l}
v=\ell(x, v, w), \quad v \in \mathbb{R}^{q} \\
u=g(x, v, w)
\end{array}\right.
$$

such that

(i) For any $(x(0), v(0)) \in \mathcal{P}_{t}$ and $w(0)=0$ we have $x(k) \rightarrow 0$ and $v(k) \rightarrow 0$ as $k \rightarrow \infty$.

(ii) For any $(x(0), v(0)) \in \mathscr{P}_{l}$, and for any $w(0) \in \mathcal{W}_{0}$ the solution of the closed-loop system satisfies

(a) $z(k) \in \&$ for all $k \geqslant 0$.

(b) $\lim _{k \rightarrow \infty} e(k)=0$.

(iii) For any $(x(0), v(0)) \in \mathscr{P}_{t}$, and $w(0) \in \mathcal{W}_{0}$, whenever we set $w(k) \equiv 0$ for $k \geq k_{0}$ for some $k_{0}$, we have $\lim _{k \rightarrow \infty} x(k)=0$.

The next theorem states that the constrained tracking domain $\mathscr{P}_{t}$ can be as large as the constrained stabilizing domain $\mathcal{P}_{s}$. This result is in tune with the result of a recent paper [1] except that our work is suitable for tracking sinusoidal signals and not just for (asymptotically) constant reference signals. Also, our work leads to a method of constructing an appropriate regulator in case of rightinvertible constraints. 
Theorem 5.3 Consider the system $\Sigma$ in (2.3) with $E=0$ (i.e. tracking), the constraint set $\& \subset \mathbb{R}^{p}$, and a bounded set $\mathcal{W}_{0} \subset \mathbb{R}^{s}$. Assume that there exists a $\rho \in(0,1)$ such that

$$
\left(C_{z} \Pi+D_{z} \Gamma\right) w(k) \in(1-\rho) s
$$

for all $w(k), k \geqslant 0$, with $w(0) \in w_{0}$. Let Assumptions $4.1,4.2$, and 4.3 hold. Furthermore, for the system $\Sigma$ with $w=0$, suppose that there exists a state feedback controller that can stabilize the system $\Sigma$ with a constrained stabilizing domain $\mathscr{P}_{\boldsymbol{s}}$. Finally assume that there exists an $\varepsilon>0$ such that

$$
\Pi w(k) \in(1-\varepsilon) \mathcal{P}_{s}
$$

for all $w(k), k \geqslant 0$, with $w(0) \in \mathcal{W}_{0}$. Then, any set $\mathscr{P}_{t}$ in the interior of the closure of the set $\mathscr{P}_{s}$ is a constrained tracking domain as defined in Definition 5.2.

Remark. Note that the above theorem applies to all the categorized constraints. Also it is worth noting that we can only preserve the domain of attraction for tracking. If exogenous disturbance is acting on the system, this persistent disturbance might be able to steer the system outside of the domain of attraction. On the other hand, the extra condition (5.2) is natural because after tracking is accomplished, the state must be in the stabilizing domain.

\section{Conclusion}

When a linear system is subject to both input and state magnitude constraints, structural properties inherent in the system dictate the solvability conditions for internal stabilization as well as output regulation. The main contribution of this paper regarding discrete-time constrained systems is to identify such solvability conditions in terms of a taxonomy of constraints, viz. minimum/nonminimum phase constraints, right-invertibility/non-rightinvertibility constraints, and constraint infinite zeros being of type 1 or not. It is shown that for right-invertible constraints, the constraint infinite zeros must be of type 1 for semiglobal and global output regulation. Under the developed solvability conditions, controllers can be constructed to achieve both stabilization and output regulation, while not violating the imposed constraints. Also, a relationship between the stabilization domain of attraction and the tracking domain of attraction is established for systems which can have non-minimum phase constraints.

\section{References}

[1] F. Blanchini and S. Miani, "Any domain of attraction for a linear constrained system is a tracking domain of attraction", SIAM J. Contr. \& Opt., 38(3), 2000, pp. 971-994.

[2] E.J. Davison, "The output control of linear timeinvariant multi-variable systems with unmeasured arbitrary disturbances", IEEE Trans. Aut. Contr., 17, 1972, pp. 621-630. Correction: Vol. 20, No. 12, 1975 , pp. 824.

[3] E.J. Davison and A. Goldenberg, "The robust control of a general servomechanism problem: the servo compensator", Automatica, 11, 1975, pp. 461471 .

[4] C.A. Desoer AND Y.T. WANG, "On the minimum order of a robust servocompensator", IEEE Trans. Aut. Contr., 23(1), 1978, pp. 70-73.

[5] B.A. FrancIs, "The linear multivariable regulator problem", SIAM J. Contr. \& Opt., 15(3), 1977, pp. 486-505.

[6] B.A. Francis and W.M. Wonham, "The internal model principle for linear multivariable regulators", Appl. Math. \& Optimization, 2(2), 1975, pp. 170194.

[7] J. HaN, A. SAberi, A.A. Stoorvogel, AND P. SANNUTI, "Constrained output regulation of linear plants", in Proc. 39th CDC, Sydney, Australia, 2000, pp. 5053-5058.

[8] Z. Lin, A.A. Stoorvogel, and A. SABeri, "Output regulation for linear systems subject to input saturation", Automatica, 32(1), 1996, pp. 29-47.

[9] A. Saberi, J. Han, and A.A. Stoorvogel, "Constrained stabilization problems for linear plants", To appear in Automatica, 2002.

[10] A. Saberi, J. Han, A.A. Stoorvogel, ANd G. Shi, "Constrained stabilization problems for discretetime linear plants", Submitted for publication, 2000.

[11] A. SAberi, A.A. Stoorvogel, and P. SANnuti, Control of linear systems with regulation and input constraints, Communication and Control Engineering Series, Springer Verlag, 2000.

[12] P. SAnNuti AND A. SAbERI, "Special coordinate basis for multivariable linear systems - finite and infinite zero structure, squaring down and decoupling", Int. J. Contr., 45(5), 1987, pp. 1655-1704.

[13] A.A. Stoorvogel AND A. SABeri, "Output regulation of linear plants with actuators subject to amplitude and rate constraints", Int. J. Robust \& Nonlinear Control, 9(10), 1999, pp. 631-657. 